\title{
The utility of 3-dimensional-printed models for skull base meningioma surgery
}

\author{
Xiao-Yu Guo", Zhen-Qiang He", Hao Duan, Fu-Hua Lin, Guan-Hua Zhang, Xiang-Heng Zhang, \\ Zheng-He Chen, Ke Sai, Xiao-Bing Jiang, Zhen-Ning Wang, Tian Xie, Zhong-Ping Chen, Yong-Gao Mou \\ Department of Neurosurgery/Neuro-oncology, Sun Yat-sen University Cancer Center, State Key Laboratory of Oncology in South China, \\ Collaborative Innovation Center for Cancer Medicine, Guangzhou 510000, China \\ Contributions: (I) Conception and design: XY Guo, ZQ He; (II) Administrative support: ZP Chen, YG Mou; (III) Provision of study materials \\ or patients: H Duan, FH Lin, GH Zhang, XH Zhang; (IV) Collection and assembly of data: ZH Chen, K Sai, XB Jiang; (V) Data analysis and \\ interpretation: ZN Wang, T Xie; (VI) Manuscript writing: All authors; (VII) Final approval of manuscript: All authors. \\ "These authors contributed equally to this work. \\ Correspondence to: Yong-Gao Mou, MD, Ph.D. Department of Neurosurgery/Neuro-oncology, Sun Yat-sen University Cancer Center, State Key \\ Laboratory of Oncology in South China, Collaborative Innovation Center for Cancer Medicine, Guangzhou 510000, China. Email: mouyg@sysucc.org.cn.
}

Background: Skull base meningioma surgery is often difficult and complicated to perform. Therefore, this study aims to investigate the effectiveness of 3-dimensional (3D)-printed models of skull base meningioma in the representation of anatomical structures, the simulation of surgical plans, and patient education on surgical outcomes.

Methods: A retrospective study of 35 patients (3D group: 19 patients and non-3D group: 16 patients) with skull base meningioma was conducted. Mimics software was used to create 3D reconstructions (with the skull, blood vessels, nerves, and tumors set to different colors), and 3D solid models were printed to determine the surgical protocols and communication pathways with the patient.

Results: The 3D-printed model can visually display the relationship of different structures, including the skull, blood vessels, cranial nerves, and tumors. The surgeon should select the proper surgical approaches before surgery through the model and pay attention to protecting the important structures during the operation. According to the models, the surgeon should cut off the blood supply to the tumor to reduce intraoperative bleeding. For patients with skull base bone destruction, the skull base repair should be prepared in advance. Patients and their families should have a thorough understanding of the disease through the model, and there should be effective communication between doctors and patients.

Conclusions: The 3D-printed model of a skull base meningioma can present the structures in a detailed manner and facilitate in helping the surgeon to develop a surgical plan. At the same time, it helps patients and their families to understand the condition and the surgical plan, which is conducive to better patient education.

Keywords: 3-dimensional printing (3D printing); anatomy; meningioma; patient education; skull base surgery

Submitted Dec 03, 2019. Accepted for publication Jan 18, 2020.

doi: $10.21037 /$ atm.2020.02.28

View this article at: http://dx.doi.org/10.21037/atm.2020.02.28

\section{Introduction}

In neurosurgery, stereo vision has undergone another 2-dimensional (2D) to $3 \mathrm{D}$ transformation, and the appearance of virtual reality technology has made great advancements in the processes of stereo vision.
Furthermore, 3D-printing allows neurosurgeons to further improve their clinical capabilities (1). The application of 3D-printing technology in conjunction with neurosurgery has become increasingly mature. Recently, some studies have reported the application of $3 \mathrm{D}$-printing technology 
in neurosurgery, including anatomical learning, tumors, cerebrovascular diseases, and functional diseases $(2,3)$.

$3 \mathrm{D}$-printing promotes the development of neurosurgery. At present, cadaver specimens are becoming increasingly difficult to acquire. The 3D-printed model helps beginners to learn anatomy better. As the level of refinement of $3 \mathrm{D}$-printing increases, we can now print fine structures such as nerves, blood vessels, and even fiber bundles. However, there are currently few reports on $3 \mathrm{D}$-printing assisting complex skull base meningioma surgery.

Skull base tumors such as meningiomas and schwannomas are common with benign tumors. Most tumors have a good prognosis if they can be completely resected. However, due to the complex structure of the skull base, the tumor is most likely closely located to important nerves and blood vessels or located deep in the skull. As a result, the tumors are difficult to expose during operation, and the surgical space is small; and therefore, the risk of skull base surgery is high, the total resection rate is low, and postoperative complications tend to be higher $(4,5)$. Because of the above reasons, the $3 \mathrm{D}$-printed model can perfectly represent the anatomical position of the skull base and the relationship between the tumor and the important surrounding structures, which is very helpful for us in choosing a more suitable surgical approach and surgical methods. With the development of 3D-printing technology, the advantages of refinement and low cost make this technology increasingly widely used.

In this study, we explored the application of 3D-printing technology in relation to the surgeon's choice of surgical approach, resection methods, and patient education in skull base meningioma surgery.

\section{Methods}

\section{Patient population}

A total of 19 patients who were diagnosed with skull base meningioma underwent surgery with a $3 \mathrm{D}$-printing technique at our institution from Nov. 1st, 2016 to Feb. 28th, 2019, in Sun Yat-sen University Cancer Center (SYSUCC) (Table 1). Complex skull base meningiomas in the same period (16 patients) were compared as a control group (Table S1). The following inclusion criteria were used: (I) pathological confirmation of meningiomas; (II) the tumor was located at the base of the skull; (III) the tumor was related to the surrounding important blood vessels or nerves (wrap or push); (IV) there was an absence of other anti-tumor treatments before admission; (V) availability of complete clinical information; (VI) the same surgeon performed all the operations.

\section{Data collection}

Demographic and clinical-pathological variables, including age, gender, pathological diagnoses, tumor size, tumor location, the extent of resection, complications, function improvement, operating time, blood loss, and length of stays, were collected using an electronic medical record system. The tumor size was defined as the largest diameter measured on pretreatment enhanced T1-weighted MRI. The extent of resection was recorded as Simpson's grade. The extent of resection was determined based on a contrast-enhanced head MRI scan performed, in addition to operation notes.

\section{D-printed models}

The Mimics 3D image reconstruction software processed the image data [computed tomography (CT), magnetic resonance imaging (MRI), and CT angiography] (Materialise, Leuven, Belgium), which sorted tissues according to their grayscale and converted tissues within a certain range of grayscale into $3 \mathrm{D}$ images through the algorithm of the software. Data with the same grayscale but belonging to different tissues, such as the skull, vasculatures, tumor, and the nerve, were segmented and visualized with distinct colors. Objet350 Connex3 printer (Stratasys, Rehovot, Israel) was used in our study. The software (Objet Studio Ver. 9.2.11.6817) provided by the 3D printer was used to select colors and materials for different organs and tissues. The printed materials mainly included Support Fullcure 705, TangoPlus Fullcure 930, VeroWhitePlus FullCure 835, VeroClear FullCure 810, Objet RGD836 VeroYellow, Objet RGD851 VeroMagenta, and Objet RGD843 VeroCyan. STL (stereolithography) files saved by Mimics were inserted into the printer; elements such as texture, color, transparency, and softness for different tissues were set. During printing, the printer sprayed the materials onto the build tray layer by layer; every layer of materials was cured by UV light. After printing, the model 
Table 1 Summary of cases and surgical results

\begin{tabular}{|c|c|c|c|c|c|}
\hline Patient & Gender & Age (years) & Location & Tumor size $(\mathrm{mm})$ & Surgical results $^{\dagger}$ \\
\hline 2 & Male & 51 & Right posterior fossa & $48 \times 40$ & ॥ \\
\hline 3 & Male & 70 & Left cerebellopontine angle & $37 \times 22$ & I \\
\hline 4 & Male & 49 & Left parasellar & $52 \times 45$ & 1 \\
\hline 6 & Male & 47 & Left parasellar & $32 \times 27$ & 1 \\
\hline 7 & Female & 57 & Tuberculum sellae & $29 \times 24$ & I \\
\hline 8 & Male & 62 & Left sphenoid ridge & $61 \times 54$ & I \\
\hline 9 & Female & 52 & Anterior skull base & $53 \times 48$ & $\|$ \\
\hline 12 & Male & 56 & Right anterior clinoid process & $42 \times 38$ & II \\
\hline 13 & Female & 53 & Right middle and posterior fossae & $47 \times 38$ & IV \\
\hline 14 & Female & 78 & Right anterior-middle cranial fossa & $75 \times 47$ & IV \\
\hline 15 & Female & 55 & Anterior skull base & $17.5 \times 15$ & I \\
\hline 16 & Male & 57 & Left sphenoid ridge & $47 \times 44$ & IV \\
\hline 17 & Male & 47 & Foramen magnum & $41 \times 28$ & I \\
\hline 18 & Female & 60 & Tuberculum sellae & $35 \times 28$ & I \\
\hline 19 & Female & 51 & Right parasellar & $26 \times 18$ & I \\
\hline
\end{tabular}

${ }^{\dagger}$, Surgical results: I, Simpson grade I; II, Simpson grade II; III, Simpson grade III; IV, Simpson grade IV.

would be checked with the reconstructed image, and the extra materials were removed by washing.

\section{Model application}

The 3D-printed model was used to accurately restore the positional relationship between the skull base, nerves, blood vessels, and tumors before surgery, and to accurately determine the blood supply artery of the tumor (Figure 1) and whether important blood vessels (such as an internal carotid artery, anterior cerebral artery, middle cerebral artery, or basilar artery), nerves (such as the optic nerve, trigeminal nerve, and oculomotor nerve) are squeezed and covered by the tumor.

Combining CT, MRI, CT angiography, and 3D-printed model, before surgery allows neurosurgeons to start from the relative positions of the skull base, nerves, blood vessels, and tumors, and observe the tumor base and the scope of invasion from multiple angles and close distances. The relevant bone markers are measured, the extent of bone removal is determined, and the optimal surgical approach is designed to maximize the exposure of the tumor based on the protection of nerve tissue and the reduction of vascular injury (Figure 2). If the tumor invasion is wide, and the skull base is severely damaged, the individualized skull repair material is designed before surgery to reconstruct the skull base (Figure 3).

To encourage patients to have a more thorough understanding of the disease and treatment methods, based on the patient's clinical symptoms, we used a 3D-printed model combined with imaging examination to inform patients of the necessity of surgical treatment, surgical methods, adverse reactions, complications, and accidents, as 
A

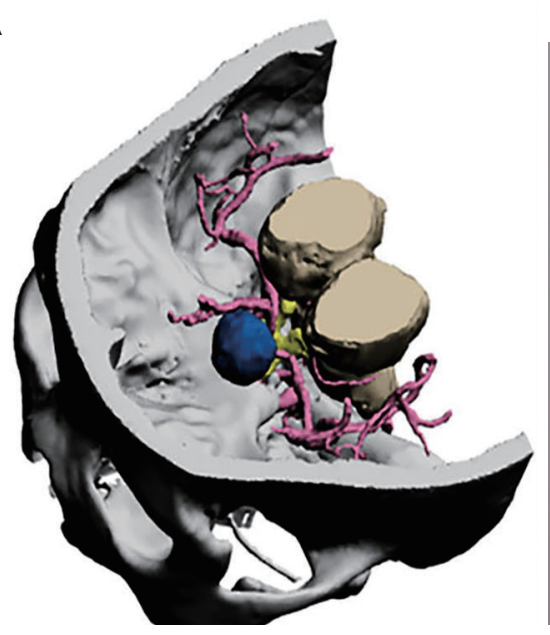

D

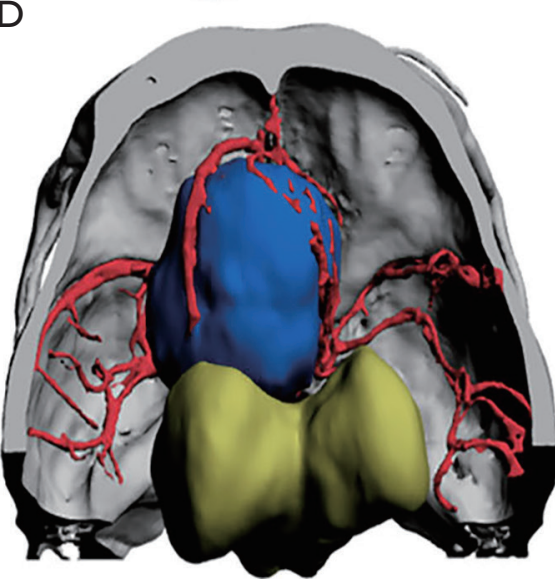

B

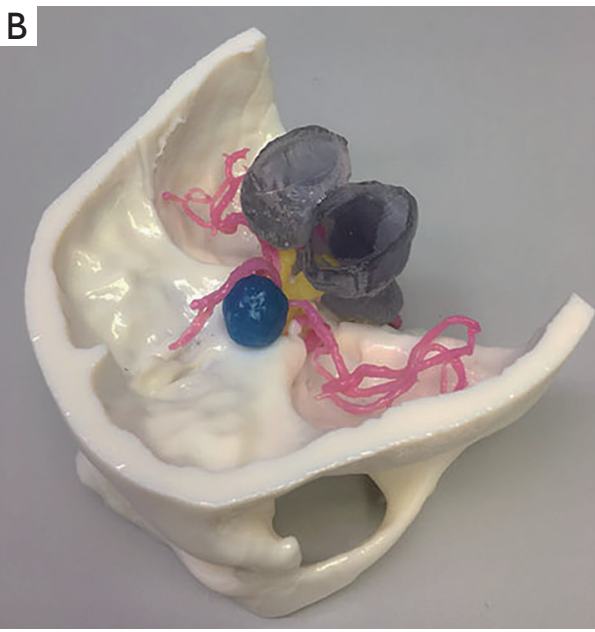

$\mathrm{E}$

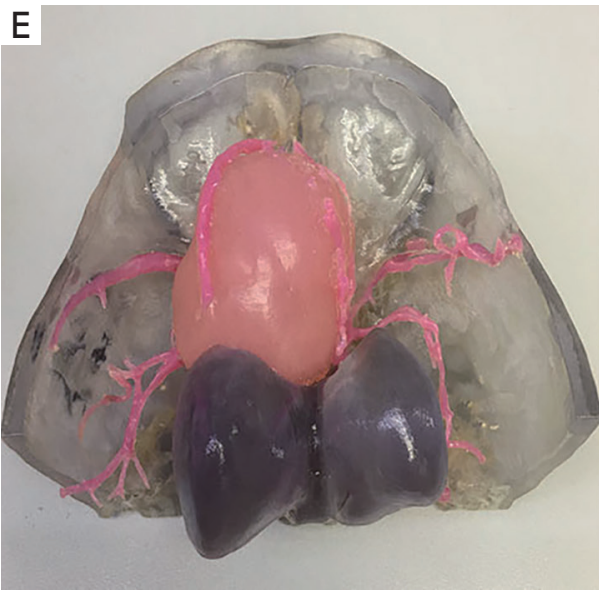

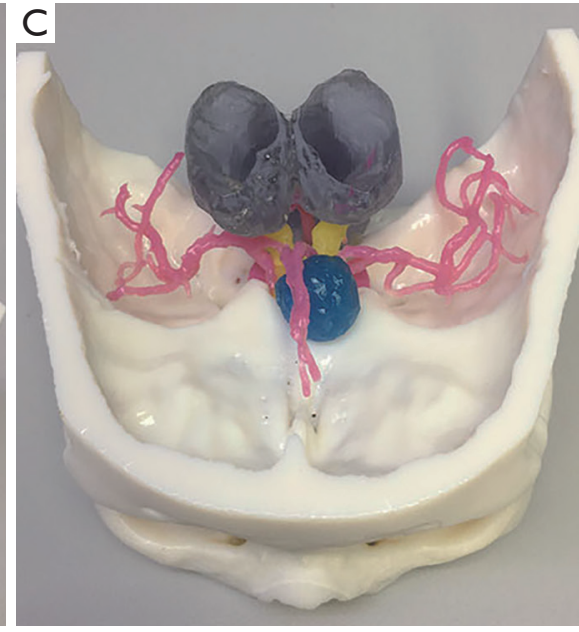



Figure 1 Screenshot of 3-dimensional (3D) images and view of the 3D-printed model. (A,D) View of 3D PDF (portable document format) files. (B,C and E,F) View of different directions of the 3D-printed model.

well as the effective measures to be taken, the prognosis of the disease, and the estimated cost (Figure 4).

\section{Statistical analysis}

Differences in baseline and clinicopathological parameters between groups were evaluated using a chi-square test or Fisher's exact test based on the type of data. All analyses were conducted using SPSS software, version 26.0 (IBM Corp., Armonk, NY, USA). P value $<0.05$ was statistically significant.

\section{Results}

There were no significant differences in our case-control studies with or without $3 \mathrm{D}$-printing-assisted surgery (Table S1). However, although there was no significant difference found in the statistical analysis, $3 \mathrm{D}$-printing technology is still one of the auxiliary methods for skull base meningioma surgery (Figures S1-S3). It is reflected in the following aspects.

\section{The surrounding tissue and relative position of skull base tumor}

In all cases, the $3 \mathrm{D}$ virtual reality image was reconstructed by software, and a $3 \mathrm{D}$-printed model was printed. All stereoscopic models clearly show the relative position of the surrounding tissue and the skull base meningiomas, and different tissues are labeled with distinct colors (Figure 1). 

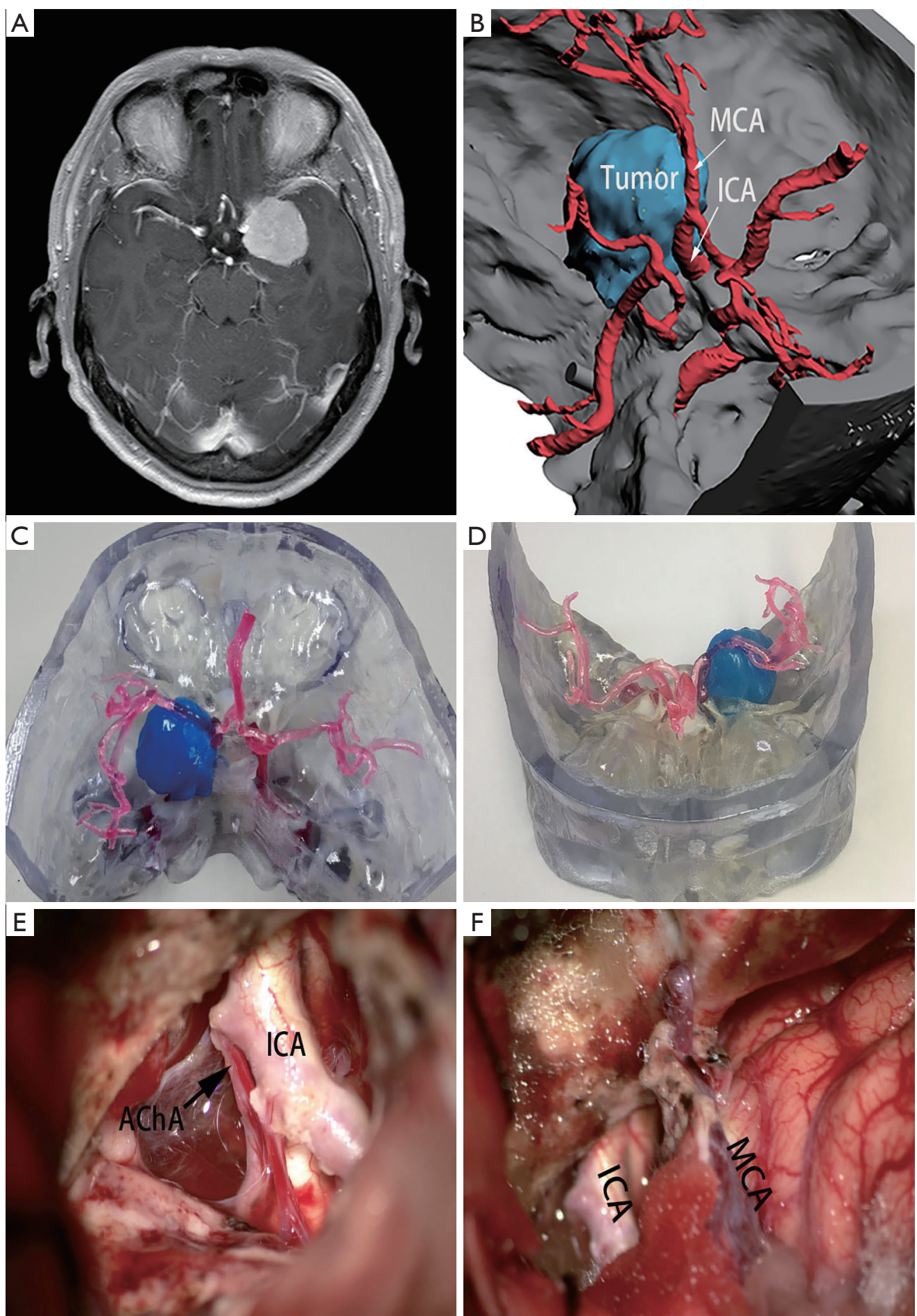

Figure 2 Protection of vital blood vessels according to 3D-printed model. (A) View of magnetic resonance imaging; (B) view of 3D PDF; (C,D) view of 3D-printed model; (E,F) final views after tumor removal, especially in terms of the preserved internal carotid artery (ICA), middle cerebral artery (MCA), and anterior choroidal artery (AChA). 



Figure 3 Individualized skull base reconstruction with 3D-printing technology. (A) 3D-printed model of bone-damaging right middle fossae meningioma; (B) design of individualized skull base reconstruction; (C) individualized skull base repair material; (D) skull base reconstruction in surgery; $(\mathrm{E})$ preoperative and $(\mathrm{F})$ postoperative appearance. 



Figure 4 Application of 3D-printed model in patient education.

During the research, to show the structure more clearly, we gradually changed from non-transparent to transparent in the use of materials. For cases in which nerves or blood vessels were contained in tumors, we used transparent materials to print tumor morphology, and the blood vessels and nerves in the tumor could be displayed (Figure 5).

\section{Surgical plan design and skull base reconstruction}

The extent of tumor exposure was determined based on the information provided by the $3 \mathrm{D}$-printed models (tumor size, neurological and vascular sacral or invasive conditions, the course of the tumor feeding artery, skull base morphology, and extent of invasion). Simulated surgery was performed on a $3 \mathrm{D}$-printed model. The degree of tumor resection and neurovascular protection under different surgical approaches were analyzed. In a patient (Figure 2), we determined the relationship between blood vessels and tumors through a $3 \mathrm{D}$-printed model. The left internal carotid artery and middle cerebral artery adhered to the tumor. We first resected part of the tumor from the lateral side of the middle cerebral artery, and then resected the remaining tumors through the medial side and protected the internal carotid and anterior choroidal arteries.

At the time of surgery, a "retrograde excision" surgical procedure was adopted. Through the path of the blood supply artery provided by the $3 \mathrm{D}$-printed model, the blood supply artery of the tumor is first cut to reduce the blood supply of the tumor, and then intertumoral decompression is performed, and finally, the tumor base is treated.

According to the $3 \mathrm{D}$-printed model, we have detailed plans for the destruction of skull base bone before surgery. We used a grinding drill to remove the hyperplastic bone. After the bone was removed, the bone was burned again by unipolar coagulation to kill the residual tumor cells. In a patient (Figure 3), individualized skull repair material was made by $3 \mathrm{D}$-printing technology before surgery. The material was used to accurately match and repair craniofacial defects to support function and appearance. After surgery, the exophthalmos was relieved, and the appearance of craniofacial was preserved. 

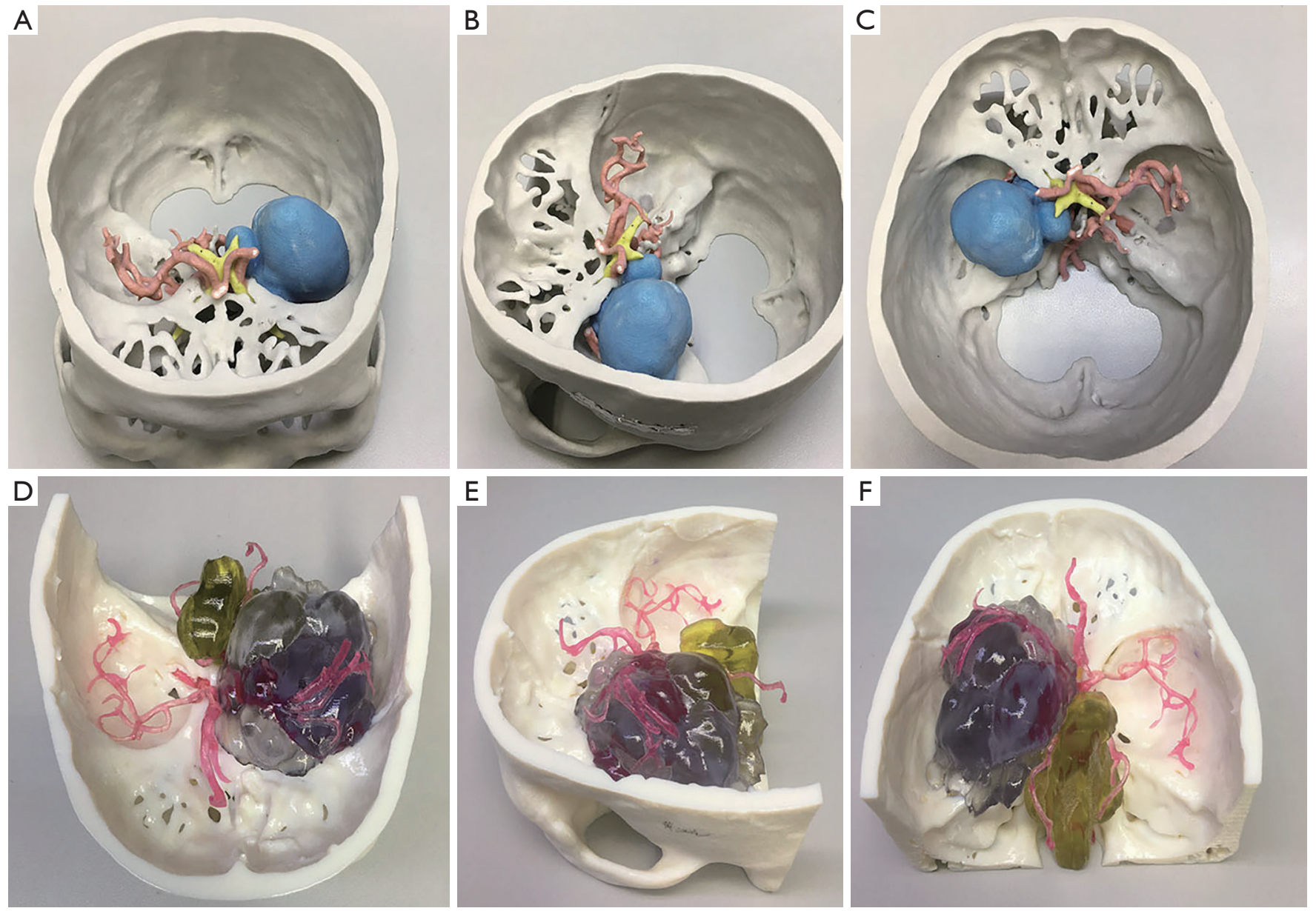

Figure 5 3D-printed model materials changed from non-transparent (A,B,C) to transparent (D,E,F).

\section{Improvement of patient education}

We communicated pre-operatively with patients according to the 3D-printed model, so that patients (especially those without medical background) could further understand the disease and realize the difficulty of surgery, the risk of surgery, and the possible complications (Figure 4). This method enhances the patient's understanding of the disease and reduces the anxiety caused by the unknown. At the same time, it makes the communication between doctors and patients more stereoscopic, visualized, shortens the communication time between doctors and patients, and improves communication efficiency.

\section{Discussion}

3D-printing technology is an emerging technology (6), and its application in the medical industry has triggered a new wave of scientific research and medical application. This technology is getting closer to improving neurosurgery, which is the most delicate and complex surgery (7). Given the importance of the brain, neurosurgery does not allow the slightest error during the process (8). Skull base surgery is one of the most difficult operations in neurosurgery (9). It has a limited operating space, which is spread over nerves and blood vessels and has higher requirements for basic knowledge and surgical skills of neurosurgeons. In our hospital, we have gained some information about using 3D-printing technology in skull base meningioma surgery.

The 3D-printed models of the skull base meningioma can truly help for restoring the patient's skull, tumor, blood vessels, and nerves. It can perfectly present the spatial relationship between the various structures and supply an anatomical basis for surgery. Based on the 3D-printed 
model, we designed the surgical approaches and performed a simulated operation on the $3 \mathrm{D}$-printed model to analyze the extent of tumor resection and neurovascular protection from each surgical approach. Preservation of nerve tissue and blood vessels must fully expose the tumor and maximize the removal of the tumor.

In this study, we used non-transparent materials to print the models in the earlier period, but these models have a great limitation in clinical practice. Therefore, we used transparent materials to print the tumor. In this way, we can more clearly see the blood vessels and nerves inside the tumor and do the best surgical projection.

At present, the method of resection of giant skull base meningioma involves intratumoral decompression and treatment of the basement alternately, but the bleeding needs to be controlled at all times during the operation. More bleeding during surgery and longer operation time are a significant risk for patients and doctors. Through the 3D-printed model, the "retrograde resection" is used to cut off the tumor supplying artery for the first time, reducing the blood supply of the entire tumor, and then performing tumor resection. During the resection process, only a small amount of oozing blood was found on the wound surface. The patient experienced less blood loss, the pressure on the surgeon was lower, the operation time was shorter, and the patient recovered faster after surgery.

A major difficulty in skull base surgery is the reconstruction of the skull base and dura mater. If the reconstruction is not complete, complications will occur, such as cerebrospinal fluid leakage, intracranial gas accumulation, brain tissue bulging, intracranial infection, etc., which can be life-threatening in severe cases. The effects of skull base reconstruction directly affect the recovery of patients after surgery (10). The widely used method currently is to use the pedicled fascia or titanium plate to rebuild the dura mater and the skull base. In this way, the likelihood of cerebrospinal fluid leakage after surgery is reduced. According to earlier studies, the reconstruction of the skull base and the dura mater can only be judged by intraoperative judgment, which is a major obstacle to a smooth operation. Hoffmann et al. (11) used a thinner CT scan and/or 3D-constructive interference in steady state (CISS) MRI or fluorescein technology to determine the extent of reconstruction, which is less accurate. We developed a skull base reconstruction plan before surgery through a $3 \mathrm{D}$-printed model. The defected bone was reconstructed with the individualized skull repair materials and fixed with titanium nails. Although many scholars believe that bone defects only affect appearance rather than function when Abe et al. (12) used a combination of bone and skin flap to repair the skull base, the patient's appearance has a great impact on the patient's social life and mental health. During the operation, it is ensured that the skull base is repaired completely to reduce the occurrence of cerebrospinal fluid leakage and intracranial infection while maintaining appearance and integrity as much as possible. Currently, bioprinting technologies use smaller fabrication units such as cells, cell aggregates, or biochemicals to produce simple tissues such as adipose tissue (13), muscles, bones, cartilage (14), 3D vasculature (15), and neural minitissues (16).

At present, most imaging examinations are $2 \mathrm{D}$ blackand-white images. It is difficult for people without a medical background to understand the meaning as they are hard to explain. Patients are only aware of the presence of tumors but do not know where the tumors are. At the same time, some medical terminology also prevents doctors and patients from communicating effectively, which is very unfavorable for the doctor-patient relationship. Patients are anxious to understand the information such as the risk of surgery and complications. To improve the situation, some research has been done. Yang et al. (17) used 3D-printed liver models to improve parental education regarding the understanding of liver anatomy and physiology, tumor characteristics, surgical procedure, and associated surgical risks. In a randomized controlled trial, Biro et al. (18) performed an analysis of the benefits of 3-dimensionally printed models in Mohs micrographic surgery (MMS). They concluded that a 3D-printed MMS model with standardized education (SE) may improve patient understanding of MMS and decrease perioperative anxiety. The brain is the most sophisticated and complex organ in the human body, and therefore the risk of performing surgery is extremely high. van de Belt et al. (19) explored the applications of $3 \mathrm{D}$-printed models in glioma patients. They assessed what patients with glioma value (or fear) about the models and found that $3 \mathrm{D}$-printed models can have a positive impact on the psychological domain of glioma patients (including coping, learning effects, and communication). In this study, we printed the skull base tumor and surrounding tissues in equal proportions. Through 3D-printed models and 2D images, we actively communicated with patients with regard to the aspects of tumor location, surgical necessity, surgical methods, surgical risks, and complications. It was easier for the patients to understand their condition better and make a prudent decision easier based on the information we provided. 3D-printed models reduce the difficulty of communication between doctors and patients, shorten the 
communication time, improve communication efficiency, and lay a foundation for further development of treatment plans.

According to our analysis, the reasons why the difference between the $3 \mathrm{D}$ group and the non-3D group is not obvious are as follows: first of all, the limitations on the number of cases in this study may lead to no significant statistical differences. Then, due to differences in tumors (size, blood supply, texture, etc.), the 3D group was more complicated than the non-3D group. The advantages of $3 \mathrm{D}$-printing cannot be reflected in the operating time, the blood loss, and the length of stays. At last, because the nerves and blood vessels in the complex skull base meningiomas were closely related to the tumor and had even been invaded, there was no significant difference between the $3 \mathrm{D}$ group and the non-3D group in terms of the number of complications and functional improvement.

There are some studies on the preoperative simulation of neurosurgery through the $3 \mathrm{D}$ digital reconstruction model. Although the perception of the anatomical structure has been improved $(20,21)$, it is clear that inspection of $3 \mathrm{D}$ data in stereo and with a sense of hand-eye coordination can give surgeons further confidence in comprehending surgical anatomies (22). According to the instruction of Objet 350 Connex 3 printer in our study, the typical deviation from STL dimensions, for models printed with rigid materials, based on size: under $100 \mathrm{~mm} \pm 100 \mu \mathrm{m}$; above $100 \mathrm{~mm} \pm$ $200 \mu \mathrm{m}$ or $\pm 0.06 \%$ of part length. It can accurately print out the solid model based on the 3D digital reconstruction model. With the advancement of this technology, although the time required for $3 \mathrm{D}$-printing is getting shorter and shorter, the time-consuming nature of $3 \mathrm{D}$-printing is also one of its limiting factors. It takes about 11-16 hours from digital reconstruction to model printing, with about 3-4 hours spent reconstructing the 3D model using the Mimics software, and the remaining time printing the model. At the same time, compared with the $3 \mathrm{D}$ digital reconstruction models, $3 \mathrm{D}$-printing models are more expensive, which is one of the reasons why it has not been popularized when it is compared to the $3 \mathrm{D}$ digital reconstruction model.

In our research, 3D-printing technology is one of the most effective auxiliary methods for skull base meningioma surgery. However, areas for continued improvement remain. Skull base meningiomas are usually closely related to the nerves and blood vessels. To ensure that the structure and function of normal tissues of patients remain, the most important thing is to improve accuracy, not only in relation to printing but also in relation to the accuracy of digital reconstruction. At the same time, the time required for printing still needs to be shortened, and the cost of $3 \mathrm{D}$-printing should also be reduced.

\section{Conclusions}

Our study proves that neurosurgeons can identify the anatomy of the skull base meningioma through a 3D-printed model and develop a surgical plan based on the model to supply individualized treatment. Therefore, $3 \mathrm{D}$-printed models can help patients to understand more thoroughly their current situation, treatment plans, and risks, reducing anxiety.

\section{Acknowledgments}

We thank AME Editing Service (http://editing.amegroups. com/) for editing the proper English language, grammar, punctuation, spelling and overall style of this manuscript. Funding: This work was supported by Science and Technology Planning Project of Guangdong Province, China (grant number 2015B010125003); Science and Technology Program of Guangzhou, China (grant number 201704020133); Science and Technology Program of Jiangmen, China (grant number 2018630100110019805) National Natural Science Foundation of China (grant number 81872324$)$. The funders had no role in study design, data collection, and analysis, decision to publish, or preparation of the manuscript.

\section{Footnote}

Conflicts of Interest: The authors have no conflicts of interest to declare.

Ethical Statement: The authors are accountable for all aspects of the work in ensuring that questions related to the accuracy or integrity of any part of the work are appropriately investigated and resolved. The study was approved by the Institutional Review Board of Sun Yatsen University Cancer Center (Guangzhou, Guangdong, China) (No. 2020-FXY-033) and adhered to the tenets of the Declaration of Helsinki.

Open Access Statement: This is an Open Access article distributed in accordance with the Creative Commons Attribution-NonCommercial-NoDerivs 4.0 International 
License (CC BY-NC-ND 4.0), which permits the noncommercial replication and distribution of the article with the strict proviso that no changes or edits are made and the original work is properly cited (including links to both the formal publication through the relevant DOI and the license). See: https://creativecommons.org/licenses/by-nc-nd/4.0/.

\section{References}

1. Rubio RR, Bonaventura RD, Kournoutas I, et al. Stereoscopy in Surgical Neuroanatomy: Past, Present, and Future. Oper Neurosurg (Hagerstown) 2020;18:105-17.

2. Lin J, Zhou Z, Guan J, et al. Using Three-Dimensional Printing to Create Individualized Cranial Nerve Models for Skull Base Tumor Surgery. World Neurosurg 2018;120:e142-52.

3. Yi X, Ding C, Xu H, et al. Three-Dimensional Printed Models in Anatomy Education of the Ventricular System: A Randomized Controlled Study. World Neurosurg 2019;125:e891-901.

4. Margalit NS, Lesser JB, Moche J, et al. Meningiomas involving the optic nerve: technical aspects and outcomes for a series of 50 patients. Neurosurgery 2003;53:523-32; discussion 532-3.

5. Lee S, Hong SH, Cho YH, et al. Anatomical Origin of Tuberculum Sellae Meningioma: Off-Midline Location and Its Clinical Implications. World Neurosurg 2016;89:552-61.

6. Aoun RJ, Hamade YJ, Zammar SG, et al. Futuristic ThreeDimensional Printing and Personalized Neurosurgery. World Neurosurg 2015;84:870-1.

7. Langridge B, Momin S, Coumbe B, et al. Systematic Review of the Use of 3-Dimensional Printing in Surgical Teaching and Assessment. J Surg Educ 2018;75:209-21.

8. Noll KR, Weinberg JS, Ziu M, et al. Neurocognitive Changes Associated With Surgical Resection of Left and Right Temporal Lobe Glioma. Neurosurgery 2015;77:777-85.

9. Diluna ML, Bulsara KR. Surgery for petroclival meningiomas: a comprehensive review of outcomes in the skull base surgery era. Skull Base 2010;20:337-42.

10. Rodrigues M, O'Malley B W Jr, Staecker H, et al. Extended pericranial flap and bone graft reconstruction in anterior skull base surgery. Otolaryngol Head Neck Surg 2004;131:69-76.

11. Hoffmann TK, Scheithauer M, Koenig R, et al. Anterior skull base defects - algorithm for reconstruction.

Laryngorhinootologie 2014;93:768-77.

12. Abe T, Goda M, Kamida T, et al. Overlapping free bone graft with galea-pericranium in reconstruction of the anterior skull base to prevent CSF leak and sequestrum formation. Acta Neurochir (Wien) 2007;149:771-5; discussion 775 .

13. Pati F, Jang J, Ha DH, et al. Printing three-dimensional tissue analogues with decellularized extracellular matrix bioink. Nat Commun. 2014;5:3935.

14. Kang HW, Lee SJ, Ko IK, et al. A 3D bioprinting system to produce human-scale tissue constructs with structural integrity. Nat Biotechnol 2016;34:312-9.

15. Kolesky DB, Truby RL, Gladman AS, et al. 3D bioprinting of vascularized, heterogeneous cell-laden tissue constructs. Adv Mater 2014;26:3124-30.

16. Gu Q, Tomaskovic-Crook E, Lozano R, et al. Functional 3D Neural Mini-Tissues from Printed Gel-Based Bioink and Human Neural Stem Cells. Adv Healthc Mater 2016;5:1429-38.

17. Yang T, Tan T, Yang J, et al. The impact of using threedimensional printed liver models for patient education. J Int Med Res 2018;46:1570-8.

18. Biro M, Kim I, Huynh A, et al. The use of 3-dimensionally printed models to optimize patient education and alleviate perioperative anxiety in Mohs micrographic surgery: A randomized controlled trial. J Am Acad Dermatol 2019;81:1339-45.

19. van de Belt TH, Nijmeijer H, Grim D, et al. PatientSpecific Actual-Size Three-Dimensional Printed Models for Patient Education in Glioma Treatment: First Experiences. World Neurosurg 2018;117:e99-105.

20. Oishi M, Fukuda M, Ishida G, et al. Presurgical simulation with advanced 3-dimensional multifusion volumetric imaging in patients with skull base tumors. Neurosurgery 2011;68:188-99; discussion 199.

21. Oishi M, Fukuda M, Ishida G, et al. Prediction of the microsurgical window for skull-base tumors by advanced three-dimensional multi-fusion volumetric imaging. Neurol Med Chir (Tokyo) 2011;51:201-7.

22. Oishi M, Fukuda M, Yajima N, et al. Interactive presurgical simulation applying advanced 3D imaging and modeling techniques for skull base and deep tumors. J Neurosurg 2013;119:94-105.

Cite this article as: Guo XY, He ZQ, Duan H, Lin FH, Zhang GH, Zhang XH, Chen ZH, Sai K, Jiang XB, Wang ZN, Xie T, Chen ZP, Mou YG. The utility of 3-dimensional-printed models for skull base meningioma surgery. Ann Transl Med 2020;8(6):370. doi: 10.21037/atm.2020.02.28 
Supplementary

Table S1 The clinicopathological features stratified by application of 3D-printing technology $(\mathrm{n}=35)$

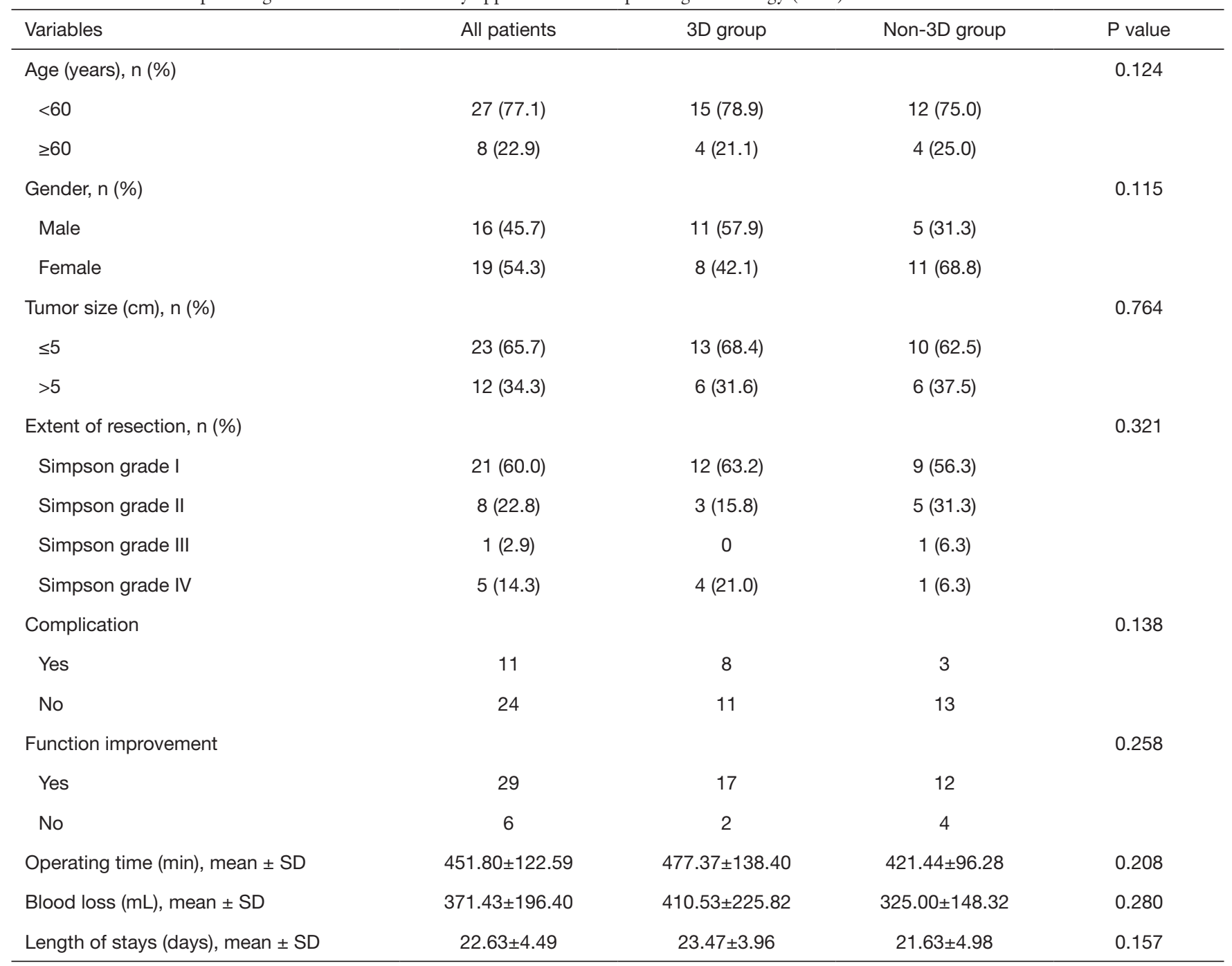

SD, standard deviation. 

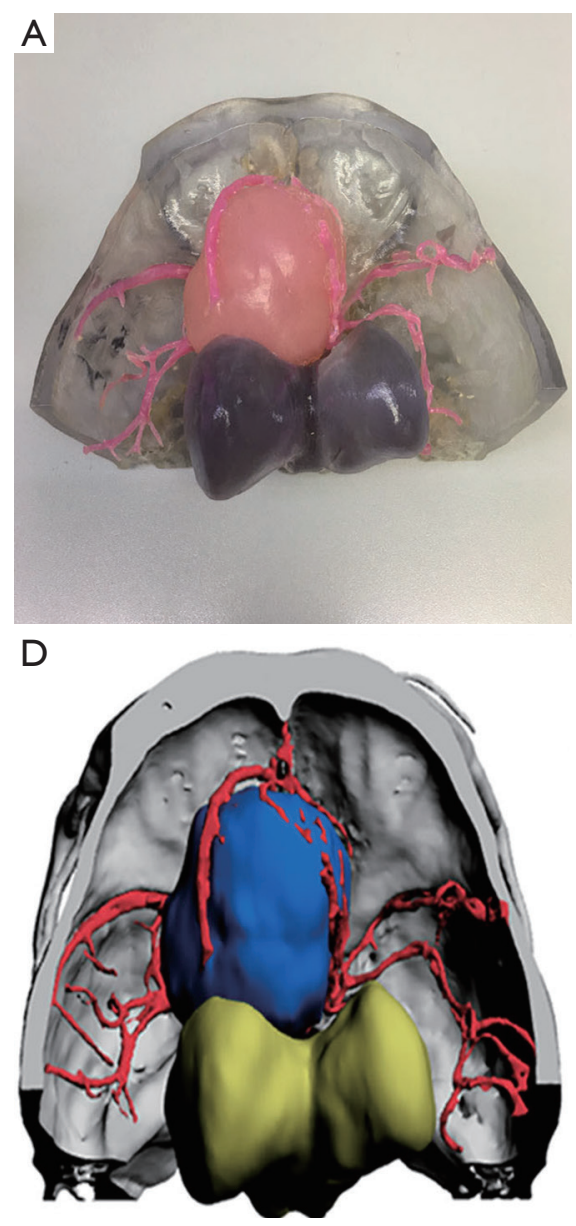

B
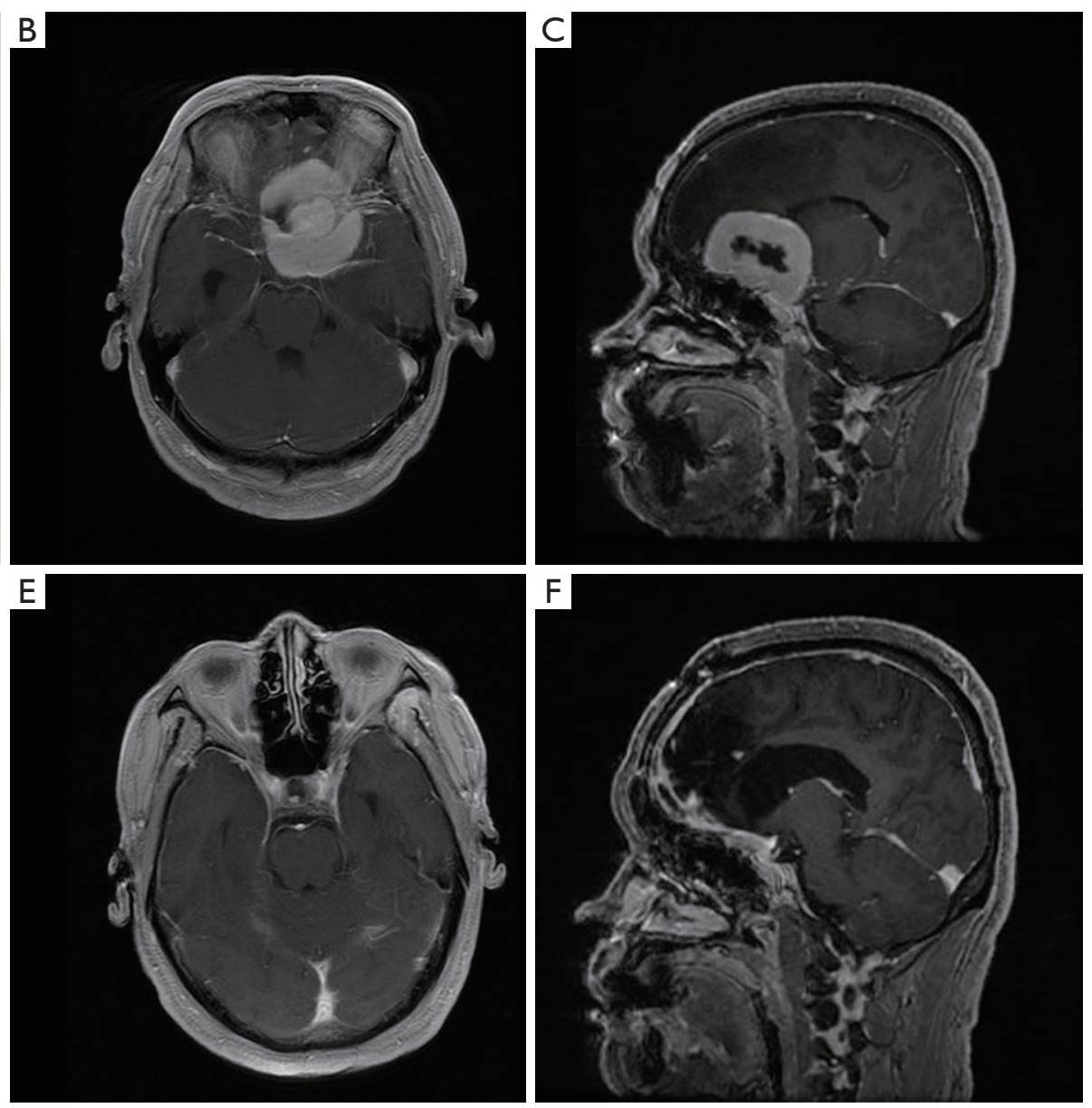

Figure S1 View of 3D-printed model (A), 3D PDF (D) and pre-operative (B,C) and post-operative (E,F) comparison of left anterior clinoid process meningioma.

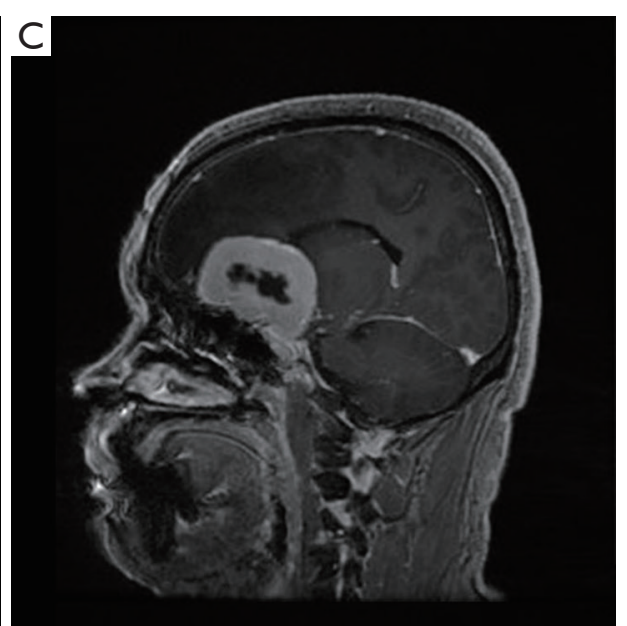

.



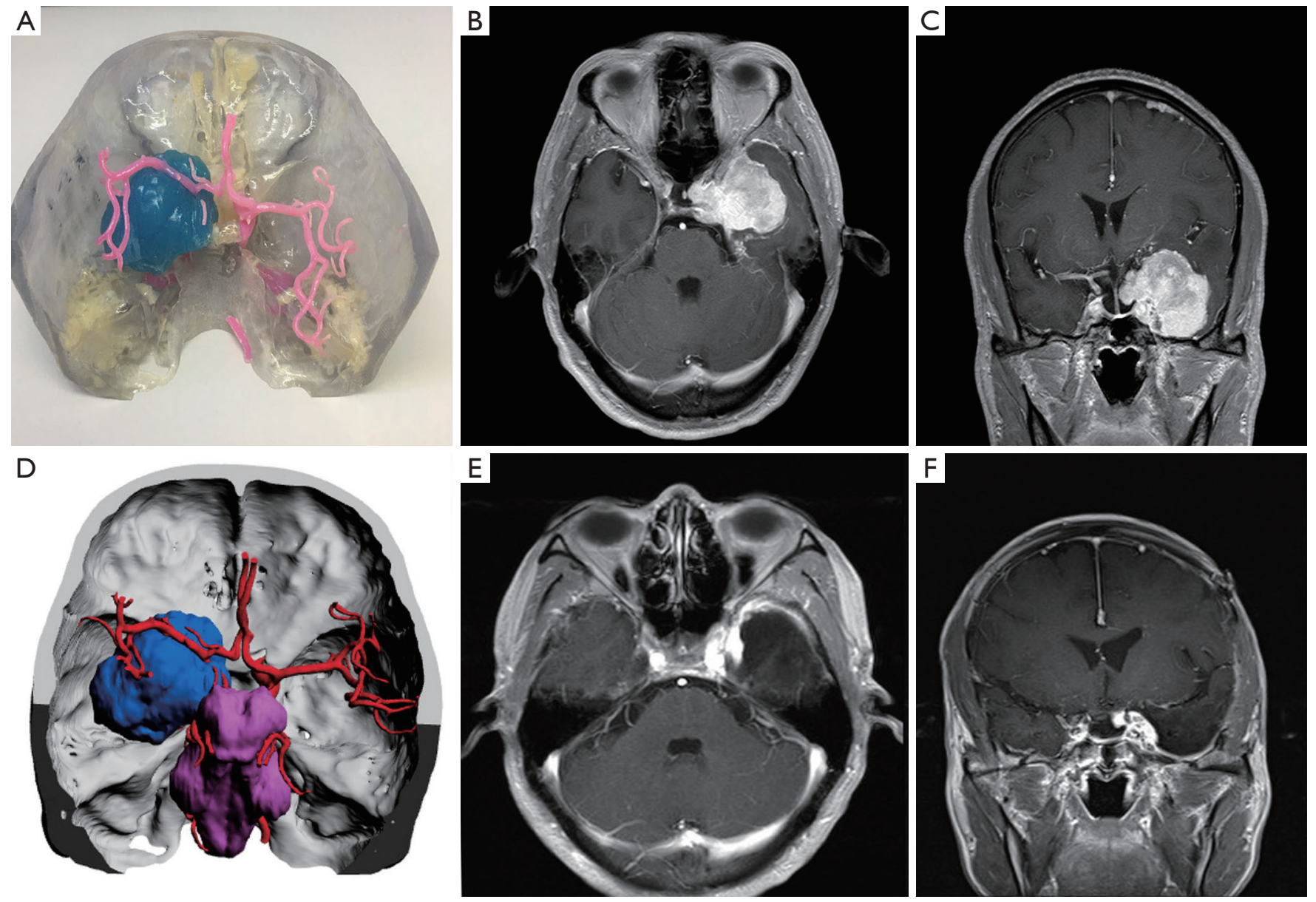

Figure S2 View of 3D-printed model (A), 3D PDF (D) and pre-operative (B,C) and post-operative (E,F) comparison of left sphenoid ridge meningioma. 

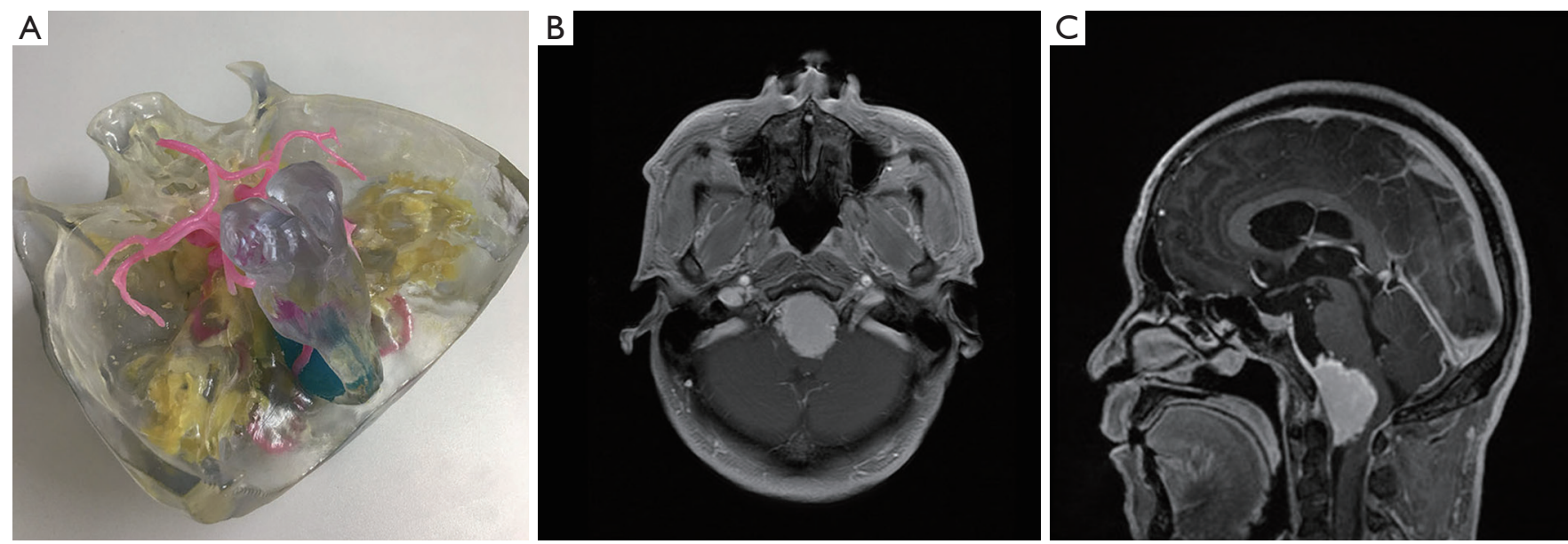

$\mathrm{D}$
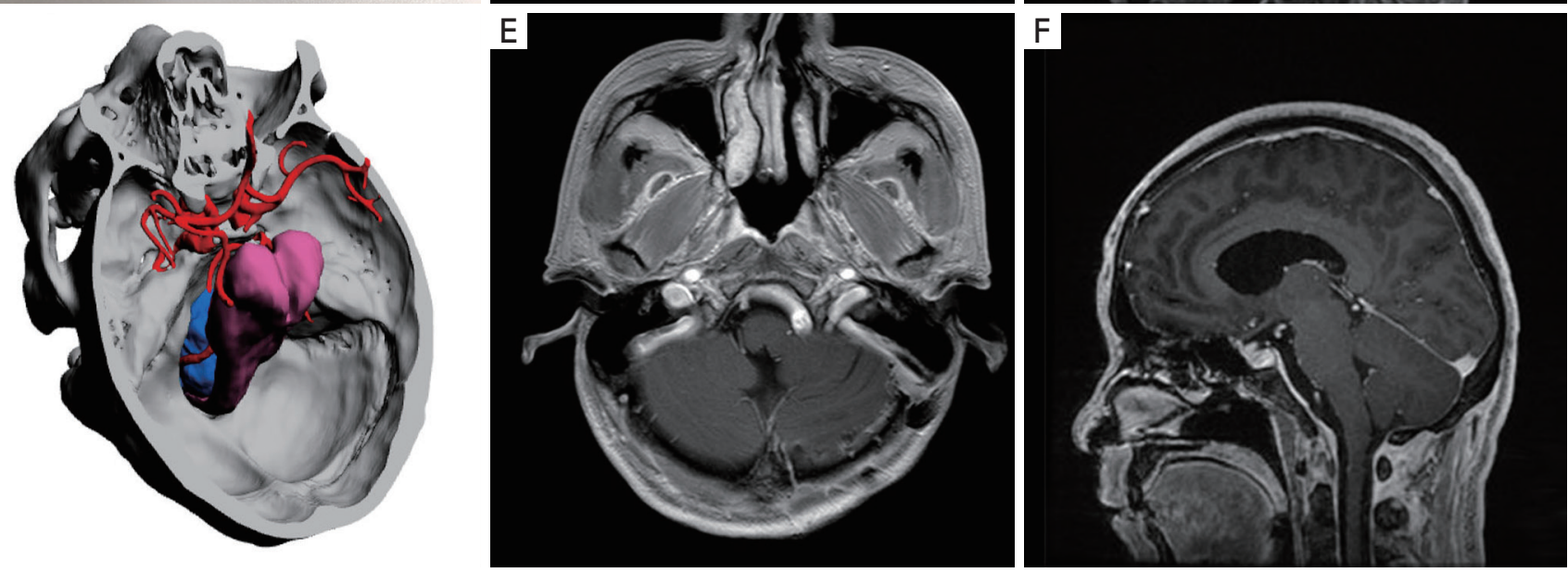

Figure S3 View of 3D-printed model (A), 3D PDF (D) and pre-operative (B,C) and post-operative (E,F) comparison of foramen magnum meningioma. 